\title{
LE LANGAGE EST UN MONDE ${ }^{1}$
}

\section{Luiz Henrique LOPES DOS SANTOS}

Universidade Federal do Rio de Janeiro

Universidade de São Paulo

Il est déjà passé le temps où la philosophie s'occupait fièrement des choses ellesmêmes. Cette constatation (d'ailleurs historiquement incontestable), le diagnostic de la situation à laquelle elle se réfère et la soumission volontaire à ses conséquences jettent les bases du programme philosophique que Francis Wolff s'est proposé d'accomplir dans Dire le monde ${ }^{2}$.

\section{Le diagnostic}

À part quelques rares voix dissonantes, les philosophes d'aujourd'hui se sont résignés à admettre qu'il est irréaliste, et même illusoire, de se proposer d'établir en quoi consiste, pour toutes les espèces de choses (réelles et idéales, matérielles et spirituelles, naturelles et surnaturelles), le fait qu'elles soient ce qu'elles sont et les raisons dernières pour lesquelles elles sont justement ce qu'elles sont. Le fond essentiel de l'être, si cela même a un sens, n'est pas à la portée d'un entendement fini. Par ailleurs, la surface factuelle de l'être est le territoire des sciences particulières, ne seraitce que parce que la philosophie, définie par sa vocation à l'universalité, ne peut pas s'ajuster au modèle d'un savoir particulier. L'antique prétendante au titre de reine des sciences ne pourrait renoncer tout bonnement à son rêve de se constituer en un savoir universel et fondateur sans perdre pour autant son droit au nom qui est le sien.

\footnotetext{
${ }^{1}$ Version étendue et modifiée de « Obra propõe-se a expandir reflexão filosófica », publié dans le journal O Estado de São Paulo (São Paulo, Brésil) le 30 mai 1998

${ }^{2}$ Wolff, F., Dire le Monde, Paris, Presses Universitaires de France, 1997.
} 
Que faire? Rien de plus simple. L'accès direct à l'essence des choses ellesmêmes lui étant interdit, pourquoi ne pas essayer une modalité d'accès oblique, susceptible de lui fournir un ersatz de l'essence des choses, qui suffirait pourtant à satisfaire ses desseins hégémoniques ? Au lieu d'adresser la question de l'essence aux choses elles-mêmes, demandons-nous désormais : que sont essentiellement les choses que nous pensons, toutes les choses que nous pensons, en tant que choses pensées?

Et voilà que, comme par un tour de passe-passe, la philosophie retrouve sa vocation. Car finalement, toute chose que nous pensons est tautologiquement une chose pensée. Aucune modalité de savoir, scientifique ou non, ordinaire ou extraordinaire, ne peut nous révéler quelque chose qui ne soit pas nécessairement, quoique obliquement, subordonné aux conditions établies par la nouvelle physionomie de l'interrogation philosophique. Aucune modalité de savoir ne peut nous révéler quelque chose qui ne soit pas inclus, ne serait-ce qu'obliquement, dans la sphère de la juridiction philosophique. Au lieu des choses elles-mêmes, la philosophie installe un univers d'entités substitutives, les choses en tant que pensées, par le truchement desquelles, désormais avec des airs de (fausse ?) modestie, elle croit maintenir dans sa zone d'influence, obliquement, la totalité des objets du savoir.

La figure dominante de la philosophie contemporaine est donc la figure d'un savoir réflexif: la tâche de la philosophie est aujourd'hui de penser ce que nécessairement sont les choses pensées par le fait même d'être des choses pensées. Or, il y a autant de chemins ouverts à l'exercice de cette réflexivité qu'il y a de dimensions de la pensée dans lesquelles nous pouvons nous installer: le langage, la conscience, l'histoire, la société, et tout ce qu'on pourra imaginer. À s'installer dans l'une de ces dimensions, le philosophe se réfugie dans un ordre substitutif, dont la forme se met à imprégner tout ce qui peut devenir objet de sa réflexion. Chacune de ces dimensions devient ainsi un ordre total, sans extérieur, un monde en somme, dans lequel la pensée philosophique s'exerce souverainement, comme un savoir universel et fondateur.

\section{Les conséquences}

De ces dimensions, le langage et la conscience sont privilégiés, en tant que dimensions immédiatement constitutives de chaque acte singulier de la pensée. Ce n'est pas un hasard si les entreprises philosophiques contemporaines les plus significatives se sont situées dans l'orbite de la philosophie de la conscience ou de la philosophie du 
langage. Le langage, cependant, a dans sa manche un atout. Il est, comme la conscience, la forme inséparable de toutes les choses pensées en tant que pensées, la forme inséparable de la présentation du monde en tant que totalité structurée ; cependant, il est le seul, en sa qualité de moyen d'interlocution, d'accord et de désaccord entre les hommes, à procurer le milieu propice pour que le monde se présente comme un ordre commun et donc objectif.

Le langage sécrète nécessairement un monde objectif et n'existe pour nous que comme un moyen de signification de ce monde. De son côté, notre monde commun est tout ce que nous pouvons dire les uns aux autres. La forme de notre langage commun est la forme même de notre monde commun, l'ordre du langage est l'ordre même de ce que nous pouvons penser, et donc dire, comme un monde. Le langage « fait monde », dit Francis Wolff. Dans les limites de ce que nous pouvons penser, le langage et le monde constituent une unité indécomposable, un langage-monde, le lieu privilégié de la réflexion philosophique. Dire le monde se propose de l'explorer.

Tout cela ne serait-il qu'un exemple de plus du linguistic turn de la philosophie contemporaine, parmi tant d'autres ? Il importe toutefois de souligner la singularité du programme. Il ne s'agit pas de dissoudre les problèmes métaphysiques, au moyen d'une distinction soigneuse entre ce qui, dans notre représentation du monde, dépend du langage et ce qui dépend du monde. C'est tout simplement impossible. Mais il ne s'agit pas non plus de décrire la structure essentielle du langage et de s'appuyer sur la solidarité de l'essence du langage et du monde, pour en conclure que cette structure est, ipso facto, la structure essentielle du monde.

En effet, nous pouvons et nous devons répondre à la question suivante : «Que doit être le monde pour qu'il soit dit par ce langage ?» Rien ne garantit pourtant, en principe, que quelque monde, un monde quelconque, puisse être tout ce qu'il devrait être pour que nous puissions le dire complètement. En disant le monde, nous présupposons et nous exigeons qu'il satisfasse aux conditions qui se trouvent impliquées dans la nature du langage qui le dit. Affirmer en principe que le monde satisfait effectivement ces conditions, toutes ces conditions et seulement elles, est pur dogmatisme.

En d'autres termes, le programme de Francis Wolff est radicalement critique. En principe, rien ne garantit que notre expérience du monde fournisse un matériau apte à répondre complètement à toutes les exigences formelles que le langage impose au 
monde qu'il sécrète. En outre, et c'est là que réside le radicalisme critique de Francis Wolff, rien ne garantit, au départ (ni à l'arrivée, finira-t-il par conclure) que ces exigences admettent, toutes en même temps, d'être complètement satisfaites par un monde quelconque. La satisfaction complète de toutes peut se révéler simplement contradictoire.

Si tel est le cas, c'est un sophisme dogmatique que d'inférer, de la corrélation essentielle entre langage et monde, que le monde est le corrélat adéquat du langage. Nous pensons et nous disons un monde, de cela (presque) personne ne doute. On peut de là conclure que ce monde, dans la mesure exacte où il est, pour nous, quelque chose de déterminé, obéit aux impératifs du langage. Réciproquement: il est, pour nous, déterminé, dans l'exacte mesure - autrement dit, ni plus ni moins - où il y obéit.

Être, c'est être déterminé, cela ne se discute pas. Dans une perspective dogmatique, les choses sont ce qu'elles sont, rien de plus, de manière absolument autosuffisante, de sorte que, pour chacune d'elles, être, c'est être complètement déterminé. Dans une perspective radicalement critique, être, c'est s'insérer dans l'ordre du langage-monde, et cela peut être une question de degré. Dans cette perspective, la détermination complète est un idéal et un impératif avant d'être un fondement inébranlable. Si la signification est un ersatz de l'essence, (une « quasi-essence », dans la terminologie de Francis Wolff) et non l'essence elle-même, c'est parce qu'elle a besoin d'être indéfiniment déterminable, mais, au contraire de l'essence, elle n'a pas besoin d'être complètement déterminée.

Entre ce qui devrait être et ce que peut être le monde de notre langage s'ouvre une marge d'inadéquation que Francis Wolff identifie comme la marge de manœuvre de la métaphysique. L'ordre de notre langage-monde ne se présente pas comme un système architectonique de complémentarité, mais comme un système dynamique de tensions. L'histoire de la métaphysique peut être lue comme une succession de tentatives, des plus raisonnables aux plus délirantes, de résolution de ces tensions, afin qu'au système puisse s'ajuster un monde qui est censé être complètement autosuffisant et déterminé.

Les résultats des efforts métaphysiques sont toujours, à des degrés divers, voués à l'échec. De fait, le dénouement dogmatique était déjà inscrit dès le premier acte : traiter le transcendantal, ce qui s'enracine dans la trame de la corrélation essentielle entre le langage et le monde, comme s'il était un attribut, absolument positif et indépendant, des choses mêmes. Cependant, il y a à apprendre de la métaphysique, de ses succès et de ses illusions. La singularité de ses constructions conceptuelles nous instruit sur la 
singularité des tensions qu'elles prétendent résoudre, la singularité de ses impasses nous enseigne à quel point ses tensions sont constitutives de notre langage-monde, et pourquoi nous ne pouvons les résoudre complètement et une fois pour toutes.

Cela signifie que le travail d'élucidation de la charpente de notre langage-monde a déjà été fait, de manière plus ou moins consciente, il y a plus de deux mille cinq cents ans. Pour que nous puissions bénéficier de ses résultats, il suffit que nous le passions au tamis de la critique, en le débarrassant de toute sa gangue dogmatique. Nous touchons ici un autre point important de la singularité de la méthode de Francis Wolff dans Dire le monde. Dans ce livre, l'histoire de la philosophie devient un élément essentiel de la méthode du philosophe, et non pas seulement négativement, en tant que dépositaire d'illusions à dissiper, mais aussi positivement, en tant qu'arsenal de vérités entrevues, dont le bon entendeur peut s'approprier, dès lors qu'il est équipé du convertisseur critique adéquat. Voyons cela de plus près.

À quelles conditions pouvons-nous dire le monde? Selon Francis Wolff, dans la Métaphysique, Aristote a déjà fait une bonne partie du travail conceptuel nécessaire pour répondre à cette question. Il y a un discours sur un monde objectif quand quelqu'un parle de quelque chose à quelqu'un (un autre ou lui-même). Si je peux parler de quelque chose et être compris, alors mon interlocuteur et moi devons non seulement employer et comprendre les mêmes symboles, mais aussi en user de façon univoque, nous devons nous accorder sur les définitions des choses dont nous parlons. Nous devons donc présupposer que les choses dont nous parlons ont, chacune, une définition (une essence, dit Aristote, dans son registre dogmatique), un ensemble de déterminations constitutives de son identité, à propos desquelles il ne peut y avoir de contradiction, puisque la contradiction, en ce cas, est le critère même de l'ambiguïté.

D'un autre côté, si, une fois nommées et définies les choses dont je parle, il reste encore quelque chose à en dire, c'est parce qu'elles sont aussi susceptibles d'une autre espèce de déterminations. Si le plus petit discours n'est pas le nom mais l'énoncé, c'est parce que les choses ne sont pas seulement ce qu'elles sont, mais qu'il leur survient aussi des événements, qui les déterminent sans toutefois endommager leur identité. Parler du monde, c'est affirmer ou nier quelque chose à propos de quelque chose à quelqu'un, capable, en principe, de nier ce qui en est affirmé ou d'affirmer ce qui en est nié. Nous pouvons parler du monde dans la mesure même où nous pouvons nous 
contredire sur ce qui arrive aux choses. Mais sur la définition des choses, nous ne pouvons pas nous contredire.

Ainsi, l'axe central de notre langage-monde, par-delà des particularités grammaticales des différentes langues, est la structure prédicative, que Francis Wolff conçoit d'une manière générale et abstraite. Nous disons le monde en nommant les choses et, par l'entrelacs de leurs noms, en énonçant ce qui leur advient. Dans ce monde, il y a des choses à nommer et des événements à prédiquer que nous appréhendons discursivement dans un ordre total et commun et que nous constituons au moyen des questionnements : "Qu'est-ce que cette chose, en ce qui la distingue des autres?»; «Pourquoi ceci arrive-t-il, au lieu de ne pas arriver?». Le langage fait monde parce que nous nommons des choses que nous présupposons définissables, et que nous énonçons des événements que nous présupposons explicables.

Depuis sa naissance, la métaphysique s'est efforcée d'imaginer des langagesmondes, dans lesquels l'un ou l'autre de ces actes aurait un pouvoir infini. Francis Wolff se propose de tirer les leçons de ces expériences fantastiques. On a parfois imaginé un monde où tout puisse être nommé et défini complètement (tout, absolument tout : chaque chose, chaque état de choses, chaque état de chaque ensemble de choses, chaque connexion d'état de choses). Dans ce cas, pourtant, savoir ce que les noms signifient (condition préalable de production d'un discours quelconque) serait déjà posséder la science totale du monde. Cette condition préalable une fois satisfaite, tout discours deviendrait vain : il n'y aurait plus rien à dire.

On a aussi parfois imaginé un monde où tout puisse être énoncé (tout, absolument tout : ce qui est et ce qui arrive). En fait, dans ce monde, tout ce qu'il y a est ce qui arrive. En lui, rien ne pourrait être reconnu comme étant le même, l'identité de la chose se désintégrerait dans le flux incessant de ses accidents instantanés, et à aucun symbole on ne pourrait attribuer quelque chose qui soit son signifié. Dans un monde où nous ne pourrions pas nous baigner, ne serait-ce qu'une seule fois, dans le même fleuve, nous ne pourrions pas non plus utiliser, ne serait-ce qu'une seule fois, un symbole dans le même sens. Dans ce monde, il n'y aurait rien à dire.

Nommer et définir, prédiquer et expliquer, sont des actes qui concourent à ce que le langage fasse monde, mais non de façon harmonieuse. Nous pouvons nommer et définir, mais nous ne pouvons pas tout prédiquer ni expliquer complètement. Et viceversa : plus nous pouvons prédiquer et expliquer, moins nous pouvons nommer et moins complètes seront nos définitions. Notre langage-monde est hybride par nature, sa 
cohésion interne résulte de la composition précaire de vecteurs qui sont en même temps complémentaires et opposés.

C'est dans l'effort pour conceptualiser dogmatiquement le centre de gravité de cette balance transcendantale que se sont forgées, selon Francis Wolff, la plupart des plus importantes notions métaphysiques - Dieu, le temps, la substance, le fondement. Leur rôle est de résoudre, autant que possible, et en même temps de masquer, autant que nécessaire, la tension dont se nourrit notre langage-monde - tension que la conversion critique de ces notions permet de mettre au jour.

Les relations entre les différents présupposés qui soutiennent notre langagemonde ne sont pas moins compliquées dans le domaine du discours pratique, dont la forme nucléaire est celle des énoncés qui imputent des actions à des personnes. Par la force de leur structure prédicative, de tels énoncés doivent présenter les actions comme des événements et les personnes comme des choses. Cependant, les actions d'une personne ne lui arrivent pas comme leur sang circule. Par définition, une action est un événement causé par la chose qu'elle concerne, une personne est une chose qui cause des événements qui la concernent. Mais ces définitions, dit Francis Wolff, semblent nous mener à une impasse.

De fait, si la cause de l'action remonte à ce qui constitue la singularité de la personne dont on dit qu'elle agit, alors l'action n'est pas quelque chose qui arrive à cette personne, mais le simple développement de sa définition - et donc ce n'est pas vraiment une action. Si Leibniz a raison, si l'acte de franchir le Rubicon est contenu dans la nature individuelle de César, alors franchir le Rubicon n'est pas ce que César fait, mais ce que César est. Si, par ailleurs, la cause de l'action remonte à ce qui arrive à la personne dont on dit qu'elle agit, et la cause de cet événement à un autre, et ainsi de suite à l'infini, alors cette personne n'est pas elle-même la cause de l'action, pas plus qu'elle n'est cause de la circulation de son sang - et donc elle n'est pas vraiment une personne.

Le dilemme est insoluble : le discours pratique attend de la structure prédicative plus qu'elle ne peut lui offrir. On a bien tenté, au moyen de concepts mythiques, comme ceux de « volonté », de « liberté », et d'« identité personnelle », d'édulcorer le concept de l'énoncé d'imputation de manière à le faire sembler logiquement acceptable. Inutile, conclut Francis Wolff. Si le monde du discours théorique est hybride, celui du discours 
pratique est contradictoire. Il n'y a qu'un seul monde, et il n'y a en lui que des choses et des événements.

Devons-nous alors conclure que les actions, et finalement l'éthique elle-même, sont des fictions, utiles peut-être, mais rien de plus que des fictions? Non, répond Francis Wolff. L'inconsistance des énoncés d'imputation ne fait que prouver que les agents ne sont pas des choses et que ce qu'ils font, ce ne sont pas des événements. La relation entre un agent et ses actions ne peut pas être dite par des prédications, et donc ne peut pas être dite du tout.

Mais dire le monde n'est pas la seule fonction du langage. En plus de l'exercer, et pour qu'elle puisse s'exercer, le langage doit être aussi capable d'indiquer - c'est-à-dire, pointer et montrer. C'est en indiquant (« ceci », « maintenant», « ici ») que les actes singuliers d'énonciation découpent originairement le monde en choses et événements singuliers. Et ce qui est plus important, si l'événement de mon énonciation n'indique pas que je suis celui qui énonce, s'il ne se montre pas comme une énonciation à moi, il ne se constitue même pas comme une énonciation. Si parler est plus qu'émettre des bruits, c'est que, derrière tout acte singulier d'énonciation, il y a la clause sous-jacente « je te dis que... », qui n'ajoute rien à ce que je dis, mais constitue l'énonciation comme telle et rend possible que, par son moyen, quelque chose soit dit. Cette clause nécessairement implicite, en qualité de présupposé originaire - doit pouvoir accompagner toutes les énonciations et mérite bien le titre d'«allocution transcendantale $»$.

Le mode transcendantal de présence $d u$ «je » dans toutes mes paroles fournit, selon Francis Wolff, le paradigme qui permet que je m'appréhende, de manière consistante, comme l'agent de mes actions. Je réponds de mes actes autant que je réponds de mes paroles. La relation entre mon énonciation et sa condition transcendantale, quand je dis « je marche », je la reconnais exister aussi entre la marche et celui qui marche - et celui qui marche, c'est celui qui dit « je » quand je dis que je marche.

Je peux comprendre ma marche selon le schème conceptuel de la prédication ; à ce moment, je le conçois comme un événement parmi les événements, dont il convient de chercher les causes. Mais je peux aussi la comprendre selon le paradigme indicatif de l'allocution ; à ce moment, j'indique une action et je m'indique comme son agent. Ici, la question des causes n'a tout simplement pas de sens. 
Les ingrédients kantiens de cette stratégie de préservation de l'éthique sont évidents. Il convient cependant de ne pas extrapoler les limites de son applicabilité, d'autant qu'à ces ingrédients s'en mêlent d'autres, d'origine bergsonienne. Elle ne permet pas, par exemple, de fonder l'idée d'une morale universelle. Je me constitue comme sujet éthique non par ma soumission à un impératif catégorique, mais par ma disposition à formuler un « interrogatif éthique » : «Est-ce que je consens à m'inscrire dans le monde comme un agent? » Cette question, je ne peux la formuler qu'à propos de moi, et moi seul je peux y répondre, parce que je suis seul à pouvoir la comprendre.

Si je suis un agent, je ne le suis pas en tant que chose, mais en tant qu'indiqué par le pronom «je» dans les allocutions transcendantales qui conditionnent mes énonciations. S'il n'y a que moi qui puisse ainsi m'indiquer, alors ni toi ni lui ne peuvent être appréhendés par moi comme responsable d'actions. L'agent n'est pas la personne métaphysique, mais la première personne grammaticale. Sa substitution par une autre quelconque le convertit immédiatement en sujet logique, simple chose, et l'action en simple événement. Sur cette substitution indue reposent les paralogismes qui maintiennent vive l'illusion métaphysique selon laquelle les énoncés d'imputation doivent avoir un sens.

L'éthique existe mais il n'existe pas de discours pratique. Il n'y a qu'une catégorie de discours, celui qui dit le monde et m'indique comme sa condition transcendantale. (On ne peut résister à la tentation de voir dans le jeune Wittgenstein le protagoniste non nommé du scénario qui aboutit à cette conclusion). Dans sa dimension objective, tout discours obéit au principe de contradiction, dans sa dimension réflexive, tout discours repose sur le présupposé transcendantal selon lequel «je parle». Ce qu'Aristote et Descartes ont cru être les premiers principes métaphysiques de l'être et de la connaissance deviennent, après la critique de Francis Wolff, les conditions les plus fondamentales de possibilité du langage.

On ne peut pas dire que Francis Wolff n'ait pas accompli son programme. Il est clair qu'il reste encore beaucoup à discuter avant de nous tenir pour satisfaits. Voyons un exemple. Selon la philosophie transcendantale de Dire le Monde, il n'y a pas de sens à demander si la forme du monde s'ajuste à la forme du langage, tout simplement parce que les conditions formelles de possibilité de notre discours sur ce qui appartient au monde sont, en même temps, les conditions formelles de possibilité d'appartenance à ce 
monde. Or, notre langage est bien notre langage. Y aurait-il donc autant de mondes possibles qu'il y a des langages possibles?

La philosophie critique de Dire le Monde veut rester aussi loin du relativisme que du dogmatisme. Pour éviter le relativisme, Francis Wolff introduit la distinction entre les caractéristiques formelles particulières des plusieurs langues, qui font les différences parmi elles, et les caractéristiques formelles générales que toute langue doit avoir pour être capable de dire et communiquer quoi que ce soit. Autrement dit, il introduit la distinction entre les caractéristiques accidentelles des langues et les caractéristiques essentielles du langage en tant que tel, et il ne fait dépendre la forme du monde commun à tous que de l'essence du langage - l'unique essence de tout langage possible et imaginable. Un lecteur moins confiant pourrait se demander : l'auteur, après nous avoir exhorté, par tous les moyens, à éviter l'illusion métaphysique de l'essentialisme, aurait-il fini, hélas, par succomber lui-même à la tentation de l'essence ?

Autre exemple: selon Wolff, si je dis d'autrui qu'il a fait ceci ou cela, mon énoncé à la troisième personne, parce qu'il est à la troisième personne, relie un fait à une chose, plutôt qu'une action à un agent. À vrai dire, il n'y a d'énoncé d'imputation qu'à la première personne. Ainsi, l'épuration critique du concept dogmatique du discours pratique paraît déboucher sur une éthique irrémédiablement solipsiste, à côté de laquelle il n'y aurait place que pour une morale positiviste. Wolff doit bien convenir que c'est le genre de conclusion que l'on ne tire qu'avec une certaine gêne. Serait-elle vraiment inévitable?

Il reste en somme des problèmes peut-être aussi difficiles que ceux que Francis Wolff s'est proposé de résoudre dans Dire le Monde. Tant mieux, parce que c'est à sa capacité à se créer des problèmes que l'on reconnaît un bon philosophe. 